“C 2020 IEEE. Personal use of this material is permitted. Permission from IEEE must be obtained for all other uses, in any current or future media, including reprinting/republishing this material for advertising or promotional purposes, creating new collective works, for resale or redistribution to servers or lists, or reuse of any copyrighted component of this work in other works."

\title{
Measurement of Dynamic Voltage Variation Effect on Instrument Transformers for Power Grid Applications
}

\author{
G. Crotti, D. Giordano \\ Istituto Nazionale di Ricerca \\ Metrologica (INRIM), \\ Torino, Italy \\ g.crotti@inrim.it, \\ d.giordano@inrim.it
}

\author{
A. Delle Femine, D. Gallo, \\ C. Landi, M. Luiso \\ Dept. of Engineering, \\ University of Campania \\ "Luigi Vanvitelli", \\ Aversa (CE), Italy \\ mario.luiso@unicampania.it
}

\author{
P.S. Letizia \\ Politecnico di Torino, \\ Istituto Nazionale di Ricerca \\ Metrologica (INRIM), \\ Torino, Italy \\ p.letizia@inrim.it
}

\author{
L. Barbieri, P. Mazza, \\ D. Palladini \\ Ricerca sul Sistema \\ Energetico \\ (RSE) \\ Milano, Italy \\ paolo.mazza@rse-web.it
}

\begin{abstract}
Within the framework of distribution and transmission grids, knowledge of Instrument Transformers (ITs) behavior in distorted conditions is a topics of great interest. Its relevance stems from the ITs wide use in metering, protection, monitoring and control applications, where their role is to reduce voltage and current to levels compatible with measuring instruments input. In force standards require that the performance of measuring instruments is assessed under realistic conditions. On the contrary, performance test of ITs are generally carried out only at rated conditions, so that their behavior under actual waveforms is not fully known. To cover this gap and starting from the hardware used for the Medium Voltage (MV) Voltage Transformer (VT) frequency characterization, a suitable setup is developed for the traceable test of VTs under a quite large set of static and time varying test waveforms. In the paper, after a short description of the setup, examples of its applications to the evaluation of the performances of ITs under different test voltage waveforms are given, including a realistic reproduction of a PQ event and focusing on the amplitude and phase modulation tests, as those suggested for the Phasor Measurement Units in IEEE Std. C37.118.1. Preliminary experimental results are shown.
\end{abstract}

Keywords-Instrument transformers, power grid, power quality, phasor measurement unit, uncertainty

\section{INTRODUCTION}

Power Quality (PQ) measurement instruments, Phasor Measurement Units (PMUs) and all metering and protection instruments installed in the distribution and transmission grids require the measurement of grid voltage and current [1]. Since in transmission and distribution grid amplitude these quantities span from hundreds of volt and tens of ampere up to hundreds of kilovolt and tens of kiloampere, transducers have to be introduced in the measurement chain in order to reduce the signals to be acquired to the input levels of the installed measurement instruments.

By examining the standards relating to the most common measuring instruments present on the power network, it can be observed that verification of their performance is required under different operating conditions and several measurement test points. For example, as regards the PQ instruments the IEC 62586-2 standard [2] requires tests performed in a wide amplitude range, from $5 \%$ to $150 \%$ of the nominal voltage, and additional frequency test points besides the power frequency ones are indicated. Furthermore, these instruments must be tested with several distorted waveforms, for example multitone signals (both harmonic and interharmonic are required), typical time-varying PQ disturbance signals (f.i. dips and swells) and also signals combining several disturbances. indications.

The IEEE C37.118.1 standard [3] and its amendment [4], focused on synchrophasor measurement methods and performance verification of PMUs, deal, among the others, with amplitude and phase modulation, frequency ramp, harmonic distortion and prescribe some tests to verify the performance of PMUs in their presence.

Even if in the power grids these measuring instruments are always coupled to Instrument Transformers (ITs), currently there is a gap in the standards about IT testing, since the accuracy verification is prescribed just at power frequency or at higher frequency but with reduced amplitudes.

Nowadays, several new kinds of voltage and current instrument transformers (active or passive, generally referred to as Low Power Instrument Transformers, LPIT) are available [5], but inductive voltage and current transformers (VTs and CTs) are still widely employed. The metrological performances of these sensors in presence of distorted signals significantly 
depend on their operating principles [6]-[11]. It has been highlighted that inductive ITs have, in particular, an intrinsic non-linear behaviour involving uncertainty increasing up to some percent, when they are used to measure harmonic disturbances [12][13]. However, the behavior of the inductive ITs in presence of non-sinusoidal and/or time varying disturbances is still not fully addressed and methods for the performance verification of ITs, to quantify the errors they can introduce in the measurement chain have to be identified. To this end, a set up has been developed at INRIM, which is designed to traceable characterize Voltage Instrument Transformers (VT) under a realistic set of test waveforms at rated voltage level. In particular, this setup can generate the waveforms prescribed by the IEEE C37.118.1 standard [3] for PMUs dynamic compliance verification as well as reproduce time-varying $\mathrm{PQ}$ disturbance. In the paper, after a short description of the setup, example of its applications to the evaluation of the performances of ITs under different test voltage waveforms are given, with focus on the amplitude and phase modulation tests, as those suggested for Phasor Measurement Units in IEEE Std. C37.118.1, and preliminary experimental results are shown.

The paper is organized as follows: signal test definitions and test procedure that can be generated by the set-up are described in Section II. Section III briefly recall the measurement setup. Preliminary experimental results of the characterization of commercial VTs are shown in Section IV. Finally, Section V draws the conclusions.

\section{WAVEFORM DEFINITIONS AND TESTING PROCEDURE}

\section{A. Signal Definition}

The developed generation system is able to produce several test waveforms, compliant with standards for PMUs and PQs performance verification (multitone, frequency ramp, dips and swells, etc.). It also allows the generation of waveforms extracted from database or synthesized by the user.

As a first example of application, the reproduction of a voltage dip disturbance in a Medium Voltage (MV) grid is addressed. To this end, a fault has been simulated to reproduce those included in the QuEEN (Quality of Electric Energy) webportal, where PQ data measured by a widespread monitoring system including 400 measurement unit installed in HV/MV substations [13] distributed all over the Italian territory are reported.

To this end, a detailed model of the distribution network used (Fig. 1). The model includes a Primary Substation (PS), modelled through sinusoidal three phase $132 \mathrm{kV}, 50 \mathrm{~Hz}$ generator and a $40 \mathrm{MVA} \mathrm{HV/MV} \mathrm{power} \mathrm{transformer} \mathrm{with} 2300$ MVA short circuit impedance. From the PS MV bus-bar, six feeders starts. The feeders include four underground cables and two overhead lines with $5 \mathrm{~km}$ to $25 \mathrm{~km}$ length. At the top of each line, a three-phase circuit breaker (CB) is installed and passive loads are places connected to some lines. A fault is simulated by closure of a $\mathrm{CB}$ on a suitable resistor.

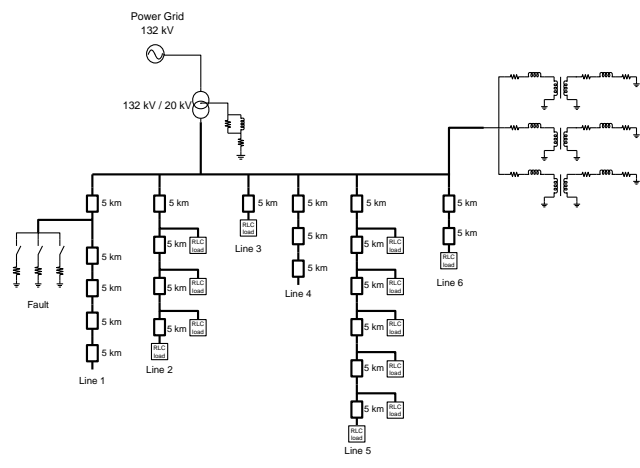

Fig. 1. Model of the considered MH/MV system.

As regards the identification of test signals under dynamic conditions, modulated signal based on [3] are considered. They consist of a fundamental tone at power frequency (i.e. the carrier), amplitude and phase modulated, according to the following expressions:

$$
\begin{aligned}
& v(t)=\sqrt{2} X_{m}\left(1+k_{x} \cos \left(2 \pi f_{m} t\right)\right) \cdot \cos (2 \pi f t) \\
& v(t)=\sqrt{2} X_{m} \cdot \cos \left(2 \pi f t+k_{a} \cdot \cos \left(2 \pi f_{m} t-\pi\right)\right.
\end{aligned}
$$

where $X_{m}$ and $f$ are, respectively, the root mean square (rms) amplitude and the frequency of the fundamental tone, $k_{x}$ and $k_{a}$ are the amplitude and phase modulation factors and $f_{m}$ is the modulation frequency .

An example of amplitude modulated signal is shown in Fig. 2.

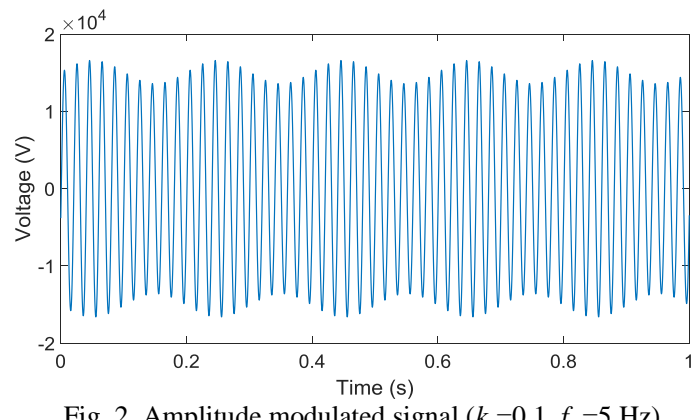

Fig. 2. Amplitude modulated signal $\left(k_{\mathrm{x}}=0.1, f_{\mathrm{m}}=5 \mathrm{~Hz}\right)$

VT accuracy is assessed according to [14], both ratio error $(\varepsilon)$ and phase error $(\varphi)$ are evaluated as:

$$
\varepsilon=\frac{k_{r} V_{s}-V_{p}}{V_{p}} \quad \varphi=\varphi_{s}-\varphi_{p}
$$

where:

- $k_{r}=V_{p, r} / V_{s, r}$ is the rated transformation ratio $\left(V_{p, r}\right.$ and $V_{s, r}$ are the rated primary and secondary voltages);

- $V_{p}$ and $V_{s}$ are the root mean square (rms) values of the primary and secondary voltage;

- $\varphi_{p}$ and $\varphi_{s}$ are phase angles of the primary and secondary voltage. 


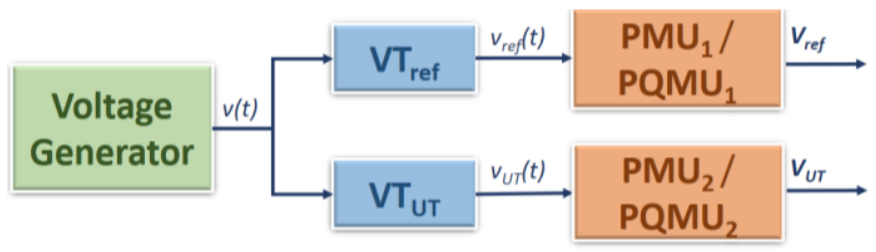

Fig. 3. Functional block diagram of the measurements

\section{A. VT Measurement Scenario}

With the aim of investigating the possible errors introduced by the VT when it is used upstream a PMU or a PQ Measurement Unit (PQMU), the measurement system configuration shown in Fig. 3 is assumed.The reference VT $\left(V T_{R E F}\right)$ and the VT under test $\left(V T_{U T}\right)$ are used to sense and reduce the same distorted voltage signal $v(t)$. Their outputs, $v_{R E F}(t)$ and $v_{U T}(t)$, are acquired and processed by two identical, and synchronized devices, which can be PQMUs or PMUs. For the PQMUs, the output quantities $V_{\mathrm{REF}}$ and $V_{\mathrm{UT}}$ are the rms voltage refreshed each half-cycle $\left(\mathrm{U}_{\mathrm{rms}}(1 / 2\right.$ cycle) $)$ at the reference and the VT chain output, from which the quantities of interest, in the considered case dip duration and residual voltage, are evaluated.

For the PMU tests, the outputs are the fundamental tone synchrophasors $\boldsymbol{V}_{\boldsymbol{R} E \boldsymbol{F}}$ and $\boldsymbol{V}_{\boldsymbol{U}}$ from which the ratio and and phase errors are evaluated, with primary and secondary voltages phasors given by:

$$
\boldsymbol{V}_{\boldsymbol{p}}=k_{R E F} \boldsymbol{V}_{\boldsymbol{R E F}} \quad \boldsymbol{V}_{\boldsymbol{s}}=\boldsymbol{V}_{\boldsymbol{U T}}
$$

where $k_{R E F}$ is the transformation ratio of $V T_{R E F}, \boldsymbol{V}_{\boldsymbol{p}}$ is the fundamental primary voltage phasor and $\boldsymbol{V}_{\boldsymbol{s}}$ is the fundamental secondary voltage phasor of $V T_{U T}$.

In addition, the Total Vector Error (TVE) is considered for the TV error analysis, as defined in [3][4]:

$$
T V E=\sqrt{\frac{\left(R e\left(k_{r} \boldsymbol{V}_{\boldsymbol{s}}\right)-\operatorname{Re}\left(\boldsymbol{V}_{\boldsymbol{p}}\right)\right)^{2}+\left(\operatorname{Im}\left(k_{\boldsymbol{r}} \boldsymbol{V}_{\boldsymbol{s}}\right)-\operatorname{Im}\left(\boldsymbol{V}_{\boldsymbol{p}}\right)\right)^{2}}{\operatorname{Re}\left(\boldsymbol{V}_{\boldsymbol{p}}\right)^{2}+\operatorname{Im}\left(\boldsymbol{V}_{\boldsymbol{p}}\right)^{2}}}
$$

If the two measuring device are assumed identical, linear and accurately synchronised, and are used to measure the same distorted voltage signal, the evaluated quantities (2), (4) and (5) can represent the errors introduced by the VT under test when it is used for syncrophasor measurement. Same consideration ca be done if the PQ event measurement is considered.

\section{MeAurement SetuP}

Starting from the hardware used for the MV VT frequency characterization, a suitable measurement setup is developed. The block diagram and the setup used for VT characterization at INRIM are shown Fig. 4a and Fig. 4b respectively.

The test waveform is generated by an Arbitrary Waveform Generator (the NI PXI 5422, 16 bit, variable output gain \pm 12 $\mathrm{V}, 200 \mathrm{MHz}$ maximum sampling rate, $256 \mathrm{MB}$ onboard memory).

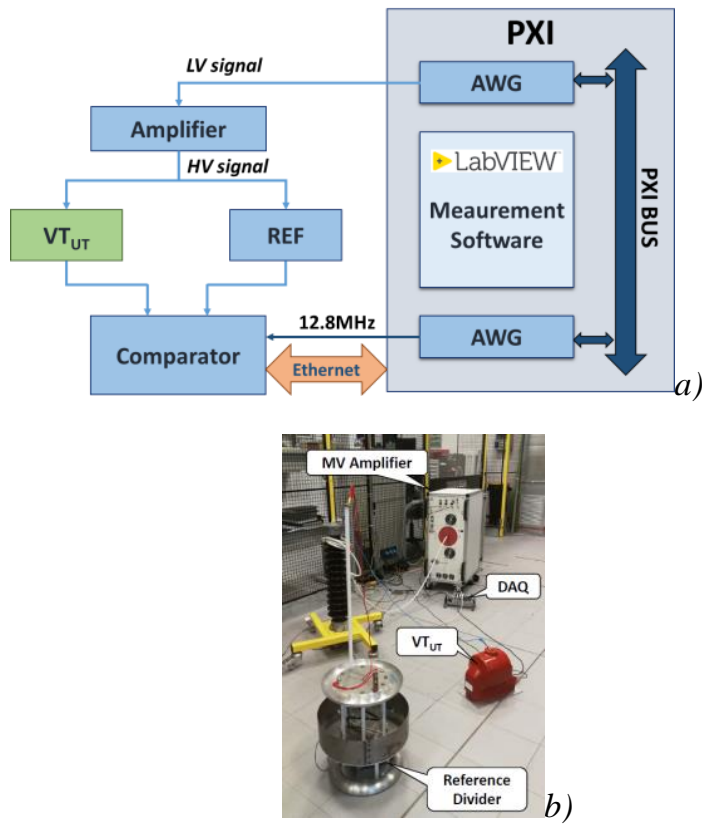

Fig. 4. Setup for the VT characterization: a) block diagram, b) experimental test bench at INRIM.

The chassis $10 \mathrm{MHz}$ PXI clock is used as reference clock for its high resolution Phase Locked Loop circuitry. The generation frequency of the AWG is therefore chosen to be an integer multiple of the generated fundamental frequency. A second AWG is used to generate a $12.8 \mathrm{MHz}$ clock, which is used as a time base clock for the signal comparator. As described in [12], The low voltage waveform from the AWG is amplified by a Trek high-voltage power amplifier $\left(30 \mathrm{kV}_{\text {peak }}\right.$, $20 \mathrm{~mA}_{\text {peak }}$ ) with wide bandwidth (from DC to $2.5 \mathrm{kHz}$ at full voltage and $30 \mathrm{kHz}$ at reduced voltages), high slew rate $(<550 \mathrm{~V} / \mu \mathrm{s})$ and low noise. Applied voltage reference values are obtained by means of a $30 \mathrm{kV}$ wideband reference divider $\left(V T_{R E F}\right.$ in Fig. 3) designed, built and characterized at INRIM.

The acquisition system is a NI cDAQ chassis with four acquisition modules: NI $9225( \pm 425 \mathrm{~V}, 24 \mathrm{bit}, 50 \mathrm{kHz}), \mathrm{NI}$ 9227 ( \pm 14 A, 24 bit, $50 \mathrm{kHz}$ ), NI 9239 ( $\pm 10 \mathrm{~V}, 24$ bit, $50 \mathrm{kHz}$ ), NI $9238( \pm 500 \mathrm{mV}, 24 \mathrm{bit}, 50 \mathrm{kHz})$. Expanded uncertainty (confidence level $95 \%$ ) is $0.007 \%$ for the ratio error and 0.07 mrad for the phase up to $1 \mathrm{kHz} 0$. The sampling clock of the digital comparator is derived from the $12.8 \mathrm{MHz}$ time base clock so that generation and acquisition are synchronized. The software for data processing and instrument control is developed in LabVIEW. A large variety of signals can be generated, such as sinusoidal, fundamental plus a harmonic tone, fundamental with $N$ harmonics, fundamental with an inter-harmonic, modulated signal, frequency ramp, transient, typical PQ events etc. The VT primary and secondary voltage are acquired with $f_{\mathrm{s}}=50 \mathrm{kHz}$ sampling frequency and $20 \mathrm{~s}$ acquisition time.

As to the PQ event test, a second comparator device (Fig. 4a) has been used. The outputs of both the reference and under test sensor are acquired by an acquisition unit that is designed by RSE to serve also as a part of a Stand-Alone Merging Unit provided with additional functions as well. This acquisition unit has two voltage channels ( $100 \mathrm{~V}_{\text {peaks }}$ or $3 \mathrm{~V}_{\text {peaks }}$ for each channel) and one current channel ( $\left.5 \mathrm{~A}_{\text {peaks }}\right)$ with 24 bit $\Delta \Sigma$ ADC and 
variable sampling frequency. The clock can be provided both internally by an on-board oscillator or by an external clock and an external TTL signal can trigger the sampling. The communication is made through an SPI / USB 2.0 Bridge interface and control and measurement software is C++ software. The prototype was characterize at RSE and INRIM and it is possible to attribute to the prototype a $110 \mathrm{~dB}$ SpuriousFree Dynamic Range (SFDR) [16].

Both PMUs and PQ algorithms are implemented through a MATLAB script. In the case of the PMU tests, because of the synchronization between generation and acquisition, the Discrete Fourier Transform (DFT) of the acquired samples is used to evaluate the voltage fundamental phasors; the observation interval is chosen equal to four cycles of the fundamental frequency and a reporting rate of $50 \mathrm{~Hz}$ is assumed.

\section{EXPERTIMAL RESULTS}

\section{A. Devices under test}

The behaviour of two different resin insulated VTs for MV phase-to-ground $50 \mathrm{~Hz}$ measurements whose rated characteristics are summarised in Table I are tested. All tests described in the following were carried out with zero burden.

Table I - VT rated characteristics

\begin{tabular}{|c|c|c|c|c|}
\hline $\mathrm{VT}$ & $V_{\mathrm{pr}}(\mathrm{kV})$ & $V_{\mathrm{sr}}(\mathrm{V})$ & Burden $(\mathrm{VA})$ & Accuracy class \\
\hline $\mathbf{A}$ & $20 / \sqrt{3}$ & $100 / \sqrt{3}$ & 50 & 0.5 \\
\hline $\mathbf{B}$ & $11 / \sqrt{3}$ & $110 / \sqrt{3}$ & 50 & 0.5 \\
\hline
\end{tabular}

\section{B. Modulated signal test}

The VTs have been tested using an amplitude (phase) modulated voltage signal with fundamental component at $50 \mathrm{~Hz}$ and rated amplitude, assuming an amplitude modulating factor of 0.1 ( $0.1 \mathrm{rad}$ for the phase), with modulating frequencies $0.1 \mathrm{~Hz}, 0.2 \mathrm{~Hz}, 0.5 \mathrm{~Hz}, 1 \mathrm{~Hz}, 2 \mathrm{~Hz}, 5 \mathrm{~Hz}$, in accordance with [3], [4].

Figs. 5 to 7, show the measured ratio and phase errors, expressed in percent and milliradians respectively, and the TVEs, in percent, as a function of the analyzed frames, corresponding to a time window of $20 \mathrm{~s}$, when an amplitude modulation is applied. Fig. 8 and Fig. 9 show the ratio error in percent and the phase error in milliradians when a phase modulation test is performed. The obtained behaviours show the presence of oscillations in the evaluated quantities, whose amplitude is quite constant with respect to the modulating being about $0.02 \%$ for the ratio error and $0.2 \mathrm{mrad}$ for the phase for amplitude modulated test. On the other hand, the number proportionally increase with the value of $f_{\mathrm{m}}$. Same behaviours, but with lower oscillation amplitudes can be observed for VT B in case of phase modulated test. These phenomena can be explained considering that the VT introduces a $50 \mathrm{~Hz}$ phase delay with respect to the reference one, which can be determined by carrying out a calibration under sinusoidal conditions, and that DFT over a $50 \mathrm{~Hz}$ cycles Under the assumption of negligible phase error of the reference sensor, no deviation will be found if the VT does not introduce any phase displacement.
Moreover, the results highlight that the peak to peak value of the oscillations is quite constant increasing the modulating frequency.

Looking at the mean value, in all test conditions, the analyzed indexes have an almost equal value and the investigated VT remains in its accuracy class.
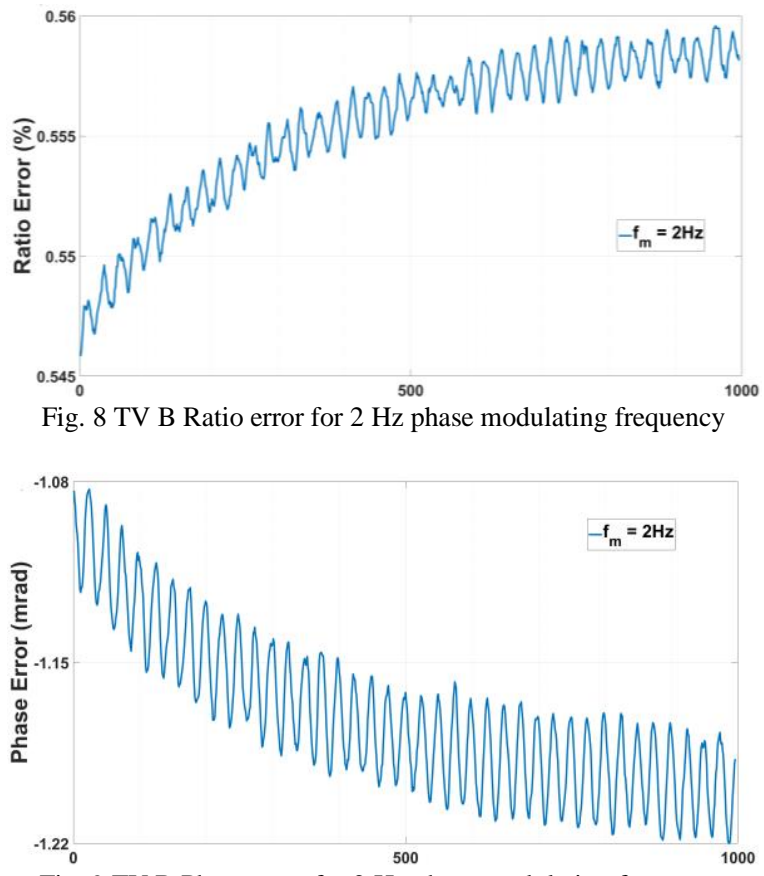

Fig. 9 TV B Phase error for $2 \mathrm{~Hz}$ phase modulating frequency

\section{A. Voltage dip test}

The voltage dips measured by the reference sensor and by the VT A under application of a simulated realistic voltage dip, are shown in Fig 10, where the voltage values are normalised to the MV grid rated primary value $(20 \mathrm{kV} / \sqrt{3})$. The evaluated quantities are the residual voltage ( $\left.U_{\text {res }}\right)$, and the voltage dip duration $t_{\mathrm{dip}}$; according to the IEC 50160, the selected dip threshold is set to $90 \%$ of the MV line rated primary voltage $U_{\mathrm{r}}$, while the hysteresis is set to $2 \% \cdot U_{\mathrm{r}}$.

As regard the voltage dip duration, since the voltage time variation of the reproduced dip is very fast, no difference is measured between the duration measured by the reference divider and the VT under test $(170 \mathrm{~ms})$. As to the measurement of the residual voltage $U_{\text {res }}$, the deviation between the value obtained with the VT and the reference one reaches a maximum difference of $0.15 \%$. This deviation is significantly lower than the quantity to be measured, but cannot be considered negligible, since it is of the same order of magnitude of the uncertainty required by the standards for the measuring instrument associated to the VT, when measuring the dip residual voltage [2]. 

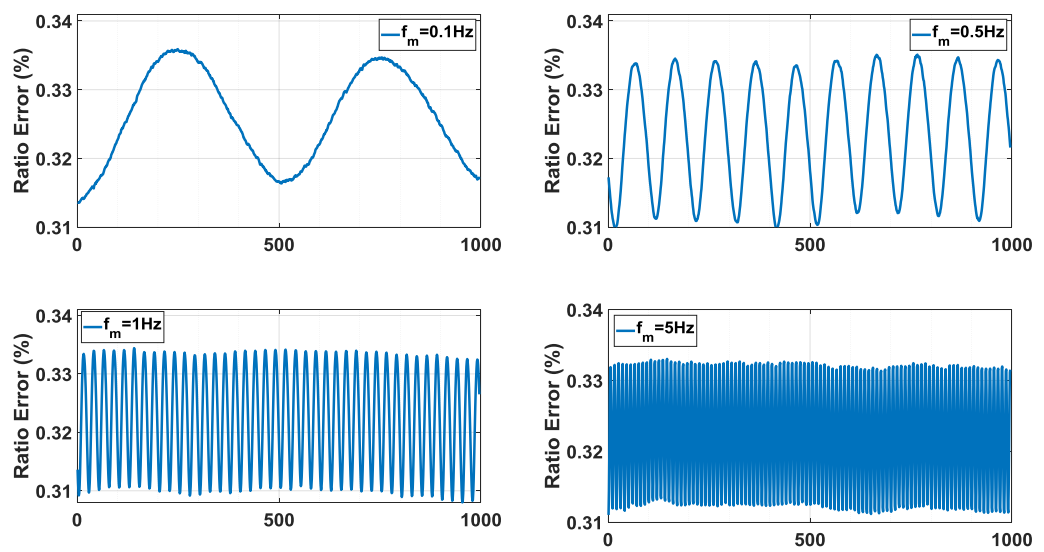

Fig. 5 TV A: Ratio error for 4 amplitude modulating frequency
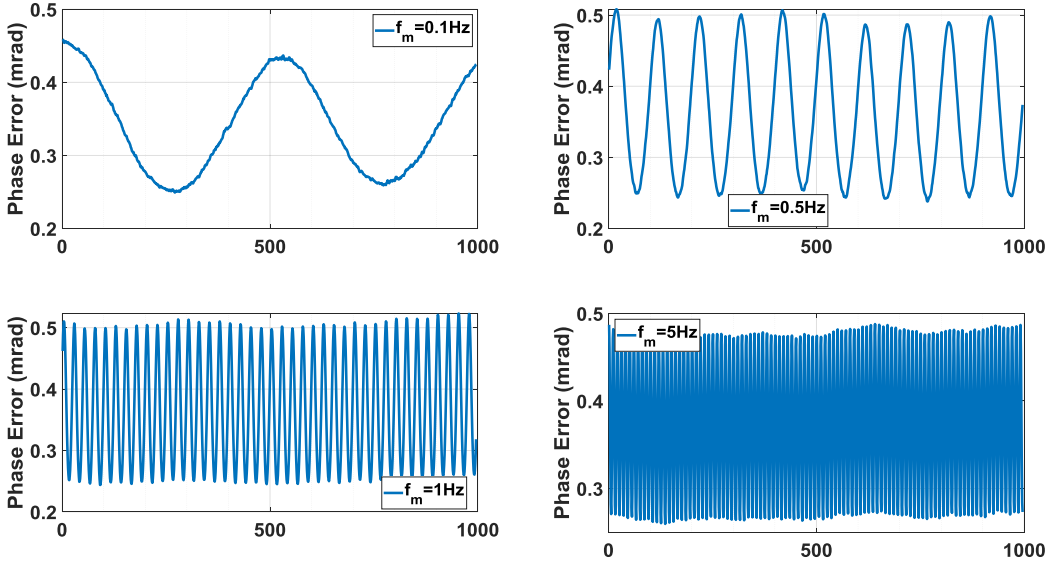

Fig. 6 TV A: Phase error for 4 amplitude modulating frequency
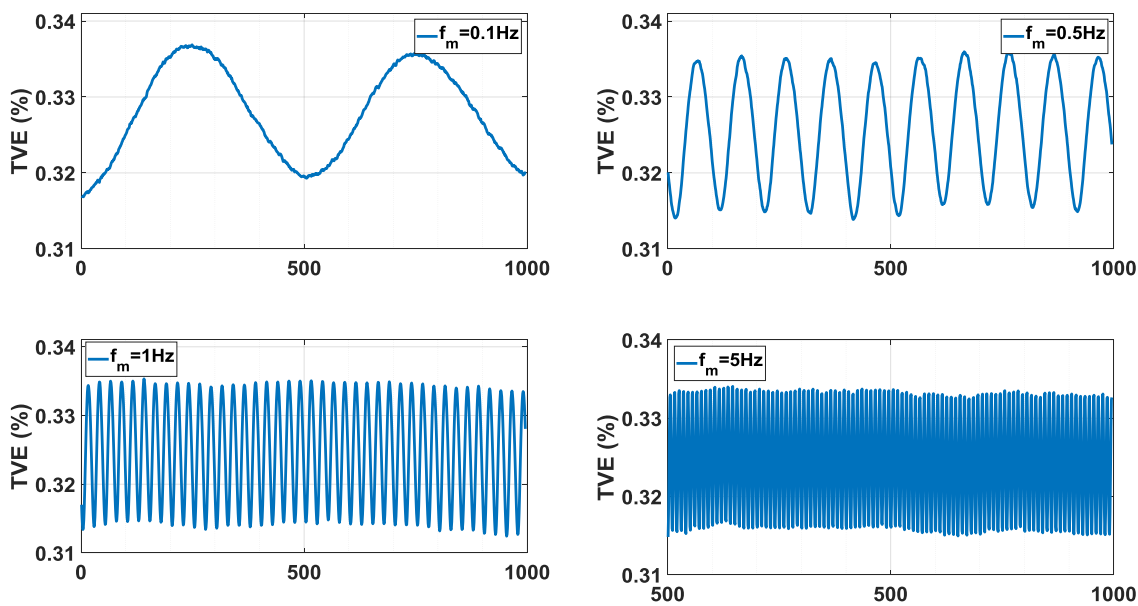

Fig. 7 TV A: Total vector errors for 4 amplitude modulating frequency 


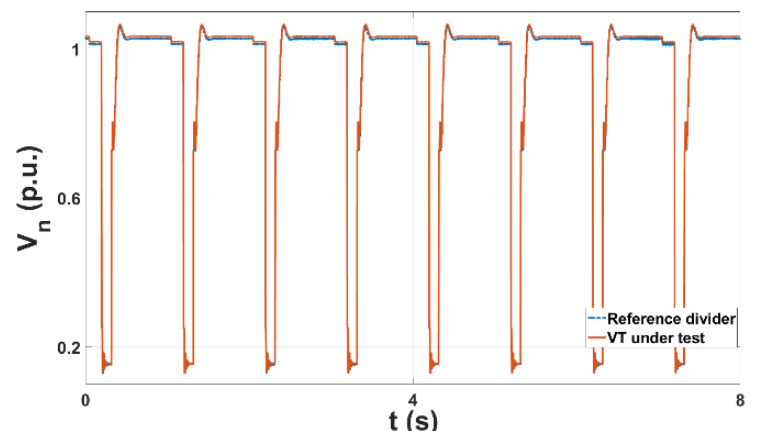

Fig.10 TV A vs Reference Divider $U_{\text {rms (1/2cycle) }}$ p.u.

\section{CONCLUSION}

A measurement approach for the quantification of the error contribution of VTs when they are involved in PQ measurement or synchrophasor measurement performed by a PMU has been presented. The developed measurement setup is modular, so it is easily extensible to characterization of different types of sensors including current transformers LPITs with analog and digital output.

First tests performed on commercial VTs for MV applications has proved the feasibility of the proposed approach. By the developed system, investigations about the VT performance will be possible, by varying other parameters, such as fundamental amplitude, frequency and phase. VTs with different rated characteristics and of different types will be also investigated.

Thanks to the large set of implemented test waveforms, the same generation and measurement system will be used to test the sensors in presence of several phenomena including typical PQ disturbances.

\section{ACKNOWLEDGEMENT}

The work presented in this paper was funded by EMPIR, 17IND06 Future Grid II project, which is jointly funded by the EMPIR participating countries within EURAMET and the European Union.

\section{REFERENCES}

[1] E. Mohns, G. Roeissle, S. Fricke and F. Pauling, "An AC Current Transformer Standard Measuring System for Power Frequencies," in IEEE Transactions on Instrumentation and Measurement, vol. 66, no. 6, pp. 1433-1440, June 2017.

[2] IEC Standard Power quality measurement in power supply systemsFunctional tests and uncertainty requirements. IEC 62586-2, 2015.

[3] IEEE standard for synchrophasor measurements for power systems. IEEE Std C37.118.1-2011, pages 1-61, Dec 2011.

[4] IEEE standard for synchrophasor measurements for power systems amendment 1: Modification of selected performance requirements. IEEE Std C37.118.1a-2014, pages 1-25, April 2014.

[5] B. Kerr, L. Peretto, N. Uzelac, and E. Scala "Integration challenges of high-accuracy LPIT into MV recloser", CIRED - Open Access Proceedings Journal, 2017(1):260-263, 2017.

[6] A. Cataliotti, D. Di Cara, A. E. Emanuel, S. Nuccio, "Improvement of Hall Effect Current Transducer Metrological Performances in the Presence of Harmonic Distortion", IEEE Trans. on Instrumentation and Measurement, vol. 59, no. 5, May 2010, pp. 1091-97.
[7] R. Stiegler, J. Meyer, J. Kilter and S. Konzelmann, "Assessment of voltage instrument transformers accuracy for harmonic measurements in transmission systems", 2016 17th International Conference on Harmonics and Quality of Power (ICHQP), Belo Horizonte, 2016, pp. 152-157.

[8] M. Faifer, R. Ottoboni, M. Prioli, and S. Toscani, "Simplified Modeling and Identification of Nonlinear Systems Under Quasi-Sinusoidal Conditions, IEEE Trans. on Instrument. and Measur., vol. 65, no. 6, June 2016.

[9] A. Cataliotti, V. Cosentino, D. Di Cara, S. Nuccio, G. Tinè, "Rogowski coil current transducer compensation method for harmonic active power error", Measurement, vol. 63, Mar. 2015, pp. 240-251.

[10] G. Crotti, A. D. Femine, D. Gallo, D. Giordano, C. Landi and M. Luiso, "Measurement of the Absolute Phase Error of Digitizers," in IEEE Transactions on Instrumentation and Measurement. doi: 10.1109/TIM.2018.2888919.

[11] A. J. Collin, A. Delle Femine, D. Gallo, R. Langella and M. Luiso, "Compensation of Current Transformers' Non-Linearities by Means of Frequency Coupling Matrices," 2018 IEEE 9th International Workshop on Applied Measurements for Power Systems (AMPS), Bologna, 2018, pp. 1-6. doi: 10.1109/AMPS.2018.8494893.

[12] G. Crotti, D. Gallo, D. Giordano, C. Landi, M. Luiso and M. Modarres, "Frequency Response of MV Voltage Transformer Under Actual Waveforms," in IEEE Transactions on Instrumentation and Measurement, vol. 66, no. 6, pp. 1146-1154, June 2017.

[13] R. Chiumeo, A. Porrino, L. Garbero, L. Tenti, M. de Nigris "The Italian Power Quality monitoring system of the MV network results of the measurements of voltage dips after 3 years campaign" CIRED - $20^{\text {th }}$ International Conference on Electricity Distribution, Prague, 8-11 June 2009 .

[14] Instrument transformers - Part 2: Additional requirements for voltage transformers. IEC 61869-3 (2013).

[15] .G Crotti; D. Gallo; D. Giordano; C. Landi; M. Luiso; "Industrial Comparator for Smart Grid Sensor Calibration," IEEE Sensors Journal, vol. 17, no. 23, pp. 7784-7793, Dec.1, 12017.

[16] P. Mazza, L. Barbieri, M. Gondola, M. Faifer, C. Laurano, R. Ottoboni, S. Toscani, M. Zanoni, "Metodologie e strumenti per la verifica e il miglioramento delle misure di tensione, corrente ed energia in Alta Tensione", (http://www.rse-web.it), March 2018. 